\title{
GAMMA-RAY LINE SPECTROSCOPY RESULTS FROM COMPTEL
}

\author{
V. SCHÖNFELDER \\ Max-Planck-Institut für extraterrestrische Physik \\ P.O. Box 1603, D-85740 Garching, Germany \\ E-mail:vos@mpe-garching.mpg.de
}

\begin{abstract}
COMPTEL aboard NASA's Compton Observatory has led to a major progress in the field of astronomical gamma-ray line spectroscopy. Highlights are the all-sky map of the $1.8 \mathrm{MeV}$ line from radioactive ${ }^{26} \mathrm{Al}$, the first detection of the $1.156 \mathrm{MeV}$ line from radioactive ${ }^{44} \mathrm{Ti}$ from a Supernova remnant (Cas A), and the detection of excessive $\mathrm{MeV}$ emission from the Orion complex that may be ascribed to excitation of ${ }^{12} \mathrm{C}$ and ${ }^{16} \mathrm{O}$ nuclei.
\end{abstract}

\section{The $1.8 \mathrm{MeV}$ All-sky Map}

As described in the paper of $\mathrm{N}$. Prantzos (these proceedings), radioactive ${ }^{26} \mathrm{Al}$ is supposed to be produced in core collapse supernovae, by hydrostatic nuclear burning in massive stars with strong stellar winds, by novae, by AGB stars or a mixture of all four. Due to its long decay time of about $10^{6}$ years, ${ }^{26} \mathrm{Al}$ traces nucleosynthesis sites over the past million years. The 1.8 $\mathrm{MeV}$ line was discovered by HEAO-C [1]. COMPTEL has now produced an all-sky map in the light of this line (see Fig. 1 [2], [3]).

The following conclusions can be derived from the map: First, the galactic plane stands out clearly. Therefore, the bulk of the observable ${ }^{26} \mathrm{Al}$ has to be of Galactic, not local origin. Second, the emission along the plane is remarkably irregular and asymmetric around the Galactic Center. There is more emission on the right side of the Galactic Center than on the left side. Substantial emission comes from regions outside the Inner Galaxy. Along the plane there are hot spots and empty regions. The reason for the clumpiness may be that nearby sources contribute significantly. Possible source candidates are: Vela, Carina, the Cygnus region, and tangential projections to the spiral arms. These findings all support our understanding that ${ }^{26} \mathrm{Al}$ is procuded in massive stars, whether these are only core-collapsed supernovae or other massive stars, is not yet clear. 


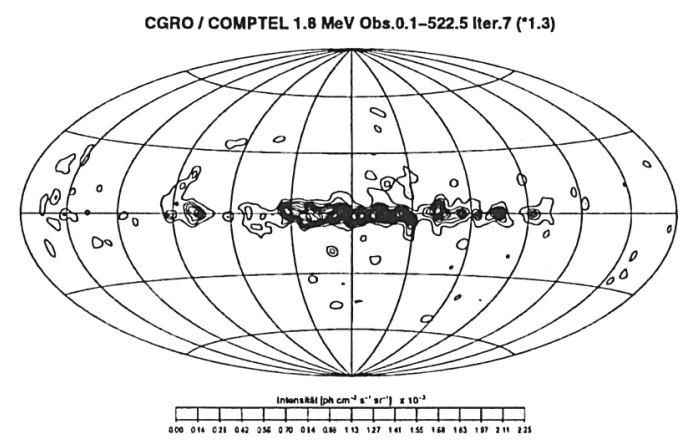

Figure 1. All-sky map in the light of the $1.809 \mathrm{MeV}$ line from radioactive ${ }^{26} \mathrm{Al}[2]$.

\section{Detections of $1.156 \mathrm{MeV}^{44} \mathrm{Ti}$ Line from Cas $\mathrm{A}$}

Cas $\mathrm{A}$ is the youngest known supernova in our Galaxy. A COMPTEL maximum-likelihood map at $1.156 \mathrm{MeV}$ from radiactive ${ }^{44} \mathrm{Ti}$ is shown in Fig. 2. If the flux measured is converted into the mass of ${ }^{44} \mathrm{Ti}$, produced at the time of the explosion (about 1670), (1 to 3) $\cdot 10^{-4} \mathrm{M}_{\odot}$ are obtained, depending on the assumed decay-time ( $\tau=78$ to 96 years) and the distance $(\sim 3 \mathrm{kpc})$, which both are not accurately known [4]. Theoretical predictions of the ${ }^{44} \mathrm{Ti}$-yield of this core-collapse SN range from a few $10^{-5}$ to $10^{-4} \mathrm{M}_{\odot}$. Since the production of $10^{-4} \mathrm{M}_{\odot}$ of ${ }^{44} \mathrm{Ti}$ should have been accompanied by about $0.1 \mathrm{M}_{\odot}$ of ${ }^{56} \mathrm{Ni}$, its decay products should have made it an optically bright shining SN. If at all, only a weak SN was seen. Flamstedt reported the detection of a 6 th magnitude star in 1680, which might have been Cas A. It now seems that Cas A was surrounded by a thick dust shell before and at the time of the explosion, which absorbed the optical light. Though the supernova shock after the event destroyed the dust, the evaporated atoms are still there, and they have been recently detected by ROSAT through their X-ray absorption [5]. This first detection of a SN-remnant in the light of the ${ }^{44} \mathrm{Ti}$-line illustrates the potential for finding previously undetected young Galactic SN by conducting a systematic line search. No convincing evidence for such events has been found in the COMPTEL data, yet [6].

\section{Gamma-ray Observations from ORION}

COMPTEL has detected excessive gamma-ray emission from the Orion complex in the 3 to $7 \mathrm{MeV}$ gamma-ray band [7]. This excess has been tentatively identified with nuclear interaction lines from excited Carbon and Oxygen nuclei. The gamma-ray emission essentially extends over the entire Orion cloud complex (consistent on a coarse scale with its CO-distribution). 


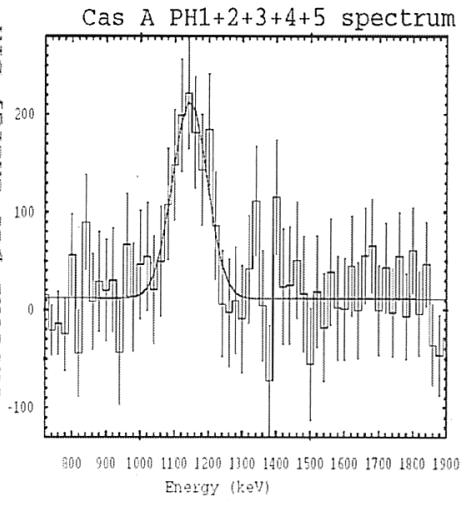

Cas A region, $\mathrm{Eg}=1.156 \mathrm{MeV}, \mathrm{PH} 1+2+3+4+5$

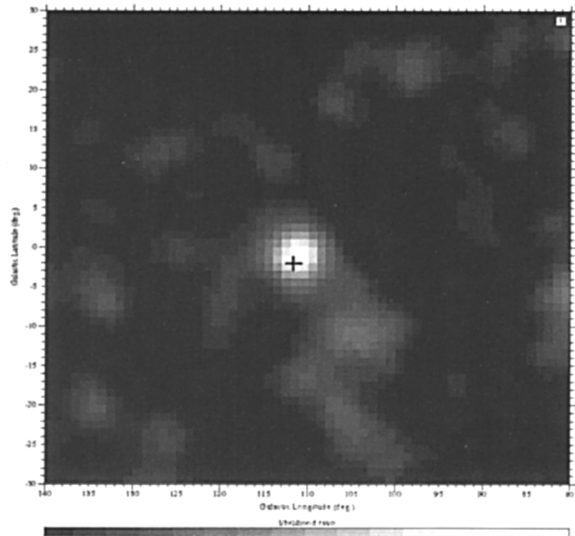

Figure 2. Detection of the $1.156 \mathrm{MeV}$ line from radioactive ${ }^{44} \mathrm{Ti}$ from Cas A. Left side: background-subtracted energy spectrum; right side: maximum-likelihood map. Data are from the sum of phase 1 to 5 from COMPTEL [4]. From the width of the measured line an expansion velocity of the remnant of $7200 \pm 2900 \mathrm{~km}$ can be inferred [4].

The 3 to $7 \mathrm{MeV}$ luminosity exceeds that at lower $(<3 \mathrm{MeV})$ and higher $(>7 \mathrm{MeV})$ energies by a factor of about 10 . The origin of the 3 to $7 \mathrm{MeV}$ excess remains unclear. The tentative identification with nuclear interaction lines is based on its spectral shape. The problems of the interpretation are manyfold: e.g. the absence of line emission below $3 \mathrm{MeV}$, which puts constraints on the chemical composition and the energy spectrum of the energetic particles producing these lines, or the high ionisation rate and the resulting short life-times and pathlengthes of the energetic particles. Recently, Parizot [8] has pointed out that it may be easier to explain the Orion gamma-ray observations by putting the source of energetic particles outside, rather than inside the Orion cloud, e.g. into the Orion-Eridanus Superbubble. Another complication of the gamma-ray line hypothesis has been pointed out by Dogiel et al. [9]. The same energetic nuclei which produce the gamma-ray lines should also produce knock-on electrons, which then may be visible via their bremsstrahlung at X-ray energies. A search in ROSAT data, performed over the entire Orion region seen in gammarays, showed no correlation between $\mathrm{X}$ - and gamma-ray line emission. The ROSAT upper limit to the 0.5 to $2 \mathrm{keV}$ X-ray luminosity is about $10^{33}$ $\mathrm{erg} / \mathrm{sec}$. This limit is close to the expected luminosity. After Ramaty et al. [10] became aware of these investigations, they pointed out that there should be two additional processes, which produce even higher X-ray fluxes. These are inverse bremsstrahlung of the energetic particles with ambient electrons, and $\mathrm{K}_{\alpha}$-line emission, stimulated by interactions with the en- 


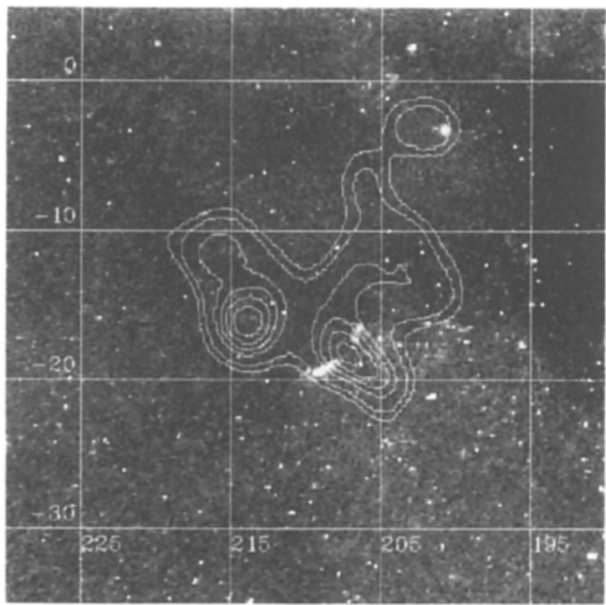

Figure 3. Superposition of ROSAT $0.5-2 \mathrm{keV} \mathrm{X-ray} \mathrm{map} \mathrm{of} \mathrm{the} \mathrm{Orion} \mathrm{region} \mathrm{onto} \mathrm{the}$ 3 to $7 \mathrm{MeV}$ intensity profiles.

ergetic particles. The sum of all three components, indeed, exceeds the ROSAT upper limit, and this might be a real problem for the gamma-ray line model [10]. One way out of this possible discrepancy might be to assume that the gamma-ray line production in Orion is not diffuse in origin, but comes from a few localised emission regions, which cannot be resolved by COMPTEL. In this case, much larger X-ray absorbing column densities might exist around these source, which would explain the non-visibility of the X-radiation [9]. Finally, it should be pointed out that the COMPTEL team considers the present Orion-results as preliminary [7]. We definitely need more data (not only at gamma-ray energies, but also at X-ray energies above $2 \mathrm{keV}$ ) and better analysis methods to substantiate the line hypothesis.

\section{References}

1. Mahoney, W.A. et al., 1984, A\&AS 120, p. 311-314

2. Oberlack, U. et al., 1996, A\&AS 120, p. 311-314

3. Oberlack, U., 1997, Thesis: Über die Natur der Galaktischen ${ }^{26}$ Al Quellen: Untersuchung des $1.8 \mathrm{MeV}$ Himmels mit COMPTEL, TU München

4. Iyudin, A. et al., 1997, Proc. of 2nd INTEGRAL Workshop, ESA-SP 382, 37-42

5. Hartmann, D. et al., 1997, Proc. of 4th Int. Conf. on Nucl. in the Cosmos Notre Dame, USA, Nucl. Phys. A.

6. Dupraz, C. et al., 1997, A\&A 324, 683

7. Bloemen, H. et al., 1997, Ap.J Letters 475, L25

8. Parizot, E.M.G., 1997, submitted to A\&A, and Proc. of 4th Compton Symp., AIP, in press

9. Dogiel, V. et al., 1997, 25th Int. Cosmic Ray Conf., Vol. 3, 133-136

10. Ramaty, R. et al., 1997, Conf. Proc. of 4th Compton Symp., AIP, in press 